\title{
Personas que viven en la calle: un problema político en construcción. Ciudad de Buenos Aires, 2007-2009
}

\section{People who live in the street: a political problem in the making. City of Buenos Aires, 2007-2009}

Martín Boy ${ }^{1}$

Aceptación: 23 agosto 2009

Aprobación: 26 abril 2010

\section{RESUMEN}

En este artículo se da cuenta de las transformaciones que se producen en la ciudad de Buenos Aires y la cristalización de ciertas manifestaciones de la pobreza que son resultado de la consolidación de una nueva cuestión social atravesada por la exclusión social y la vulnerabilidad. En este contexto, el incremento detectado de personas que viven en la calle en 1997 impulsa un conjunto de políticas sociales que convierten a esta problemática en un tema de interés político. De esta forma, es necesario dar cuenta de qué manera y bajo qué modalidades esta temática es incorporada en la agenda de la ciudad de Buenos Aires y cuáles son los aspectos que han quedado por fuera del diagnóstico inicial. Finalmente, a lo largo de este trabajo se señalan cuáles son los debates conceptuales que se están produciendo al interior de algunos de los programas del Gobierno de la Ciudad de Buenos Aires que tienen como población beneficiaria a los denominados Sin Techo.

Palabras clave: Personas en situación de calle, políticas sociales, situación habitacional.

\begin{abstract}
In this paper, there is an analysis of the transformation of the city of Buenos Aires and of the crystallization of certain poverty aspects, which are the result of the new social question characterized by social exclusion and vulnerability. In this context, the increase in the people that live on the streets in 1997 encouraged a set of social policies that transformed this issue into one of political interest. In consequence, it is necessary to consider in which way and under which forms this subject matter is added to the city's agenda and which are the aspects that have been left aside in the initial diagnosis. Finally, throughout this paper there is a specification of the conceptual debates this issue produced in the treatment of the social programmes implemented by the Government of the City of Buenos Aires that have the Sin Techo population as beneficiaries.
\end{abstract}

Key words: People living on the streets, social policy, homeless.

Becario Doctoral de la Universidad de Buenos Aires (UBA). Especialista en Planificación y Gestión de Políticas Sociales. Docente en la materia Sociología Urbana en la carrera de Sociología de la Facultad de Ciencias Sociales de la UBA. mgboy_99@yahoo.com 


\section{Introducción}

La década de los años noventa ha dejado huellas en la ciudad de Buenos Aires y en la vida cotidiana de sus habitantes. Procesos vinculados con las transformaciones estructurales han dejado profundos cambios en el tejido social. En este sentido, la retirada del pleno (o casi) empleo, el avance del mercado por sobre lo social, la aparición de la desocupación permanente y el incremento de la pobreza y la indigencia tienen como consecuencia directa el cambio del paisaje urbano de Buenos Aires.

En este artículo será de interés dar cuenta de cómo la exclusión social, la vulnerabilidad y en particular la situación de las personas que viven en la calle son incorporadas en la agenda política porteña, sin perder de vista las transformaciones estructurales antes mencionadas.

La creación de políticas públicas en el Gobierno de la Ciudad de Buenos Aires destinadas a atender a las personas que viven en la calle presentó especificidades que son interesantes de destacar: se creó un aparato conceptual para dar cuenta de esta situación, se comenzó a elaborar estadística que permita realizar un seguimiento de la población en situación de calle y se diseñaron novedosos abordajes del problema habitacional desde el escenario político.

Algunas preguntas que guiarán este trabajo serán: ¿qué sucedía en la ciudad de Buenos Aires en 1997 que motivó la creación del primer programa para personas que viven en la calle?; ¿cómo fue diagnosticada la problemática de este grupo y qué formas de atención se diseñaron?; ¿la estadística elaborada permite dar cuenta de dimensiones que los programas sociales no tienen en cuenta?

Este artículo parte del trabajo de campo realizado en la ciudad de Buenos Aires en el marco de la investigación doctoral que llevo adelante. Las principales fuentes utilizadas son los documentos que dan origen a los programas sociales (decretos e informes de gestión), estadística elaborada por diferentes organismos del gobierno porteño y los testimonios de los principales funcionarios del Gobierno de la Ciudad de Buenos Aires que se encuentran al frente de los programas y que fueron entrevistados durante el período 20072009.

\section{Transformaciones en la ciudad de Buenos Aires}

La década de los años noventa en la Argentina está directamente relacionada con la profundización de políticas de corte neoliberal que implicaron fuertes transformaciones del paisaje urbano porteño y de la vida cotidiana de sus habitantes. La flexibilización laboral, la desindustrilización y la segregación residencial son algunos de los rasgos protagonistas de una nueva ciudad que se caracteriza por la dualización social y el surgimiento de nuevos actores que representan a los grupos sociales postergados: los movimientos de desocupados, cartoneros y personas viviendo en las calles como principales exponentes.

La nueva cuestión social en la ciudad de Buenos Aires se encuentra atravesada por la pobreza y la vulnerabilidad a partir de la no tenencia de una vivienda propia y la falta de un empleo formal con sus respectivos reaseguros (Merklen 2000). En este contexto, la retirada de ciertas instituciones que en la Argentina a lo largo del siglo XX cohesionaban a la sociedad como por ejemplo el pleno empleo y la escuela pública asociada al ascenso social tienen como una de sus principales consecuencias que los sectores populares comiencen a elaborar estrategias propias a partir de "instituciones sustitutas": el barrio y la familia (Merklen, 2000). En este sentido, el retraimiento de lo público implicó que los marginados comiencen a depender cada vez más de ellos mismos.

Si las redes barriales y familiares comienzan a ser claves para elaborar estrategias de supervivencia económica, ¿qué sucede con quienes quedan desempleados y no cuentan con este capital social?

Como se mencionó anteriormente, el paisaje urbano se modificó y la presencia constante de la pobreza en la ciudad tuvo repercusiones en el escenario político. En este contexto, la situación de las personas que viven en la calle comienza a ser problematizada desde las políticas públicas del Gobierno de la Ciudad de Buenos Aires. Pero de qué forma se 
incorpora esta temática en la agenda política de la ciudad; ¿cómo es definida y delimitada la población que será asistida por los programas sociales que se crearán desde 1997 en adelante?; ¿esta población fue conceptualizada a partir de una carencia habitacional, de un problema de empleo o como manifestación de una emergencia social transitoria o permanente?; ¿cuáles son las características principales y cuáles son las innovaciones que presentan los programas que se crean?

\section{La construcción del término Sin Techo ante un cambio de perfil de la población}

En diversos países de Latinoamérica el término para referirse a las personas que viven en las calles es distinto y tienen diferentes connotaciones ${ }^{2}$ : Sem Teto en Brasil (Ferreyra, 2006), población callejera en México (Pérez García, 2008), personas en situación de calle en Chile (Habitar la calle, 2005:11), habitante de la calle en Colombia (Moreno, 2003). La pregunta que surge es: ¿cómo fue definida esta población en la Argentina y qué implicaciones se derivan?

En la ciudad de Buenos Aires el concepto que se implementó a partir de 1997 con la creación del primer programa social para quienes viven en la calle fue Sin Techo. Este concepto se encuentra vigente en la actualidad, aunque ya con fuertes críticas desde la academia y desde los propios funcionarios de los programas que conviven permanentemente con los alcances y, sobre todo, con las limitaciones de este concepto.

En Argentina el término escogido para nombrar a las personas viviendo en las calles desde el escenario político fue difiriendo a lo largo del tiempo. Según las fuentes consultadas, en las primeras décadas del siglo XX la población que vivía en la calle estaba conformada por trabajadores rurales que migraban a través de los trenes de carga de acuerdo a las temporadas de cosecha. Se los identificaba como vagabundos, locos y/o anarquistas, en un contexto generalizado de ascenso social. En 1920, se los comenzó a denominar "crotos" cuando el legislador José Camilo Crotto presentó un proyecto de ley en la Provincia de Buenos Aires para que pudieran viajar gratuitamente en los ferrocarriles (Biaggio, 2006) ${ }^{3} \mathrm{y}$, de esa forma, arriben a los campos.

Por un lado, con el desmantelamiento de la red ferroviaria, la incorporación de tecnología en el sector agropecuario a partir de la década del setenta y la consiguiente disminución de demanda de mano de obra; y, por el otro, con el incremento del desempleo, la pobreza y la indigencia, el perfil de las personas que viven en las calles fue modificándose cualitativa y cuantitativamente en los últimos quince años del siglo XX. En términos cualitativos, las personas que viven en la calle ya no responden al perfil de los trabajadores golondrinas, sino que provienen de diferentes grupos sociales que se vieron desplazados en un contexto de descenso social. Según Biaggio (2006), en la actualidad la población que vive en la calle presenta una gran diversidad: algunos llegan a esta situación a partir de desalojos; otros a partir de no poder costear las rentas de casillas ${ }^{4}$ o de cuartos de hotel; otros son cartoneros, desempleados, pacientes psiquiátricos externados que no tienen dónde y con quién vivir, personas adictas a drogas, ex reclusos y jóvenes que vivieron su infancia en hospicios, entre otros perfiles. Por otro lado, las personas que viven en las calles de la ciudad de Buenos Aires en su mayoría provienen de otras provincias del interior de Argentina e incluso de países limítrofes (más adelante, consultar Gráfico $\mathrm{N}^{\circ} 4$ ), es decir, que la migración es una variable a tener en cuenta a la hora de caracterizar a esta población.

Los cambios cualitativos que se produjeron en el perfil de la población que vive en las calles de la ciudad de Buenos Aires en un contexto de empobrecimiento y de descenso social, y su incremento cuantitativo trajo con-

\footnotetext{
2 Para más información, consultar BOY, M. (2008). "Las políticas públicas y sus definiciones sobre quienes viven en la calle: sus alcances y sus límites. El caso de la ciudad de Buenos Aires, 1997-2007". Ponencia presentada en el 1er Congreso Iberoamericano de Teoría del Habitar. Iberoamérica una forma de habitar. Facultad de Arquitectura, Urbanismo y Diseño. Universidad Nacional de San Juan, 6 al 9 de mayo.

3 BIAGGIO, M. (2006), "Linyera, ser o no ser: normas, códigos y estrategias de supervivencia de los hombres de la calle"; ponencia presentada en el VIII Congreso de Antropología Social en la Universidad Nacional de Salta.

$4 \quad$ Se entiende por casilla a las viviendas que están construidas con cartón o latón.
} 
sigo la creación de nuevos conceptos que tuvo el propósito de atender a este nuevo perfil de personas. En este sentido, el concepto de "croto" ya no parecía reflejar las características de este grupo que ya no estaba conformado por trabajadores rurales golondrinas sino por los marginados del sistema productivo.

Con la creación del programa Sin Techo en 1997 en el Gobierno de la Ciudad de Buenos Aires se instala con fuerza el concepto $\operatorname{Sin}$ Techo, definido como

"toda persona adulta que se encuentre pernoctando en espacios públicos o privados, sin contar con una infraestructura que permita ser caracterizada como vivienda precaria. Esta última supone contar con paredes y techos que otorguen privacidad, albergar pertenencias y generar una situación relativamente estable. También quien se resguarda con cartones o maderas en un bajo puente o autopista. No se considera en situación de calle a una persona que habita en una villa de emergencia u ocupa una casa tomada. Tampoco quien construye una habitación precaria, aislada, en un baldío" (Ferreira, 2001:18).

Como se puede observar, la definición de "Sin Techo" estará delimitada por la relación del individuo con lo habitacional. La persona Sin Techo queda definida a partir de la carencia total de una vivienda o una infraestructura parecida a una vivienda. De esta manera, quien pernocta en la calle se diferenciará de otros grupos marginados como por ejemplo los habitantes de "villas miseria" o de "nuevos asentamientos urbanos" ya que no cuentan ni siquiera con casillas de madera o chapa armadas alrededor de las vías de los trenes. Así es como los programas lograron definir y distinguir a sus propios beneficiarios de otros grupos empobrecidos que viven en la ciudad.

Siguiendo la definición creada desde los programas, se esperaría que la atención de esta problemática esté a cargo de organismos que tengan una trayectoria en temas habitacionales. Sin embargo, si bien la ciudad cuenta con instituciones dedicadas a las problemáticas de vivienda, la cuestión de los Sin Techo fue abordada desde el Ministerio de Desarrollo
Social del Gobierno de la Ciudad de Buenos Aires y no desde el Instituto de la Vivienda, por ejemplo. Es decir, que no ha sido incorporado en la agenda política de la ciudad de Buenos Aires como un problema habitacional, sino como un problema social que debía ser asistido pero que, paradójicamente, da nacimiento a nuevas prestaciones que pueden ser pensadas como "novedades habitacionales". Este último punto será retomado más adelante.

Los Sin Techo y la incorporación de la problemática en la agenda política porteña

Como se mencionó anteriormente, la incorporación en la agenda política de la situación de las personas que viven en las calles fue a través de la perspectiva de la asistencia social y no desde políticas de vivienda, tal como la definición de Sin Techo parecería indicar. La perspectiva de la asistencia social determinará el perfil de políticas que comenzaron a crearse en la ciudad de Buenos Aires a partir de 1997.

Las políticas para Sin Techo básicamente son cuatro y las características principales son las siguientes:

\section{Programa Sin Techo}

El Programa Sin Techo se crea en 1997 a partir de los cambios cualitativos en el perfil de esta población antes reseñados y del incremento que se observaba de personas viviendo en la calle en un contexto de aumento del desempleo, de la pobreza y de la indigencia. Este programa surge con el objetivo de lograr

“la rehabilitación/reinserción de aquellas personas que viven en la calle y que pueden ser categorizadas como "leves" $e$ "incipientes", personas que no han roto todavía sus vínculos familiares y/o comunitarios, aunque a veces hayan perdido iniciativa y esperanza de resolver sus problemas. En relación a los "crónicos graves", cuya reinserción social se hace muy difícil, el objetivo a lograr sería la detención del proceso de deterioro" (Informe preliminar, 1997).

Para lograr la anhelada rehabilitación de las personas y/o su reinserción social, desde los 
programas sociales se tiende a trabajar con las personas Sin Techo que pueden ser resociabilizables principalmente a partir de su relación con el mercado laboral y con la recuperación de las redes familiares. El equipo profesional formado por psicólogos y trabajadores sociales es el que diagnostica la situación y las posibilidades de reinserción en cada caso.

La infraestructura del Programa Sin Techo está basada en un recurso: los hogares de tránsito. En estos hogares las personas (hombres y mujeres, en forma separada) son albergadas por períodos de tiempo de alrededor de seis meses como máximo con posibilidad de ser trasladadas/os a otro hogar en caso de que siga necesitando albergarse en este tipo de dispositivos como parte del proceso encaminado hacia la reinserción social de la persona.

\section{Buenos Aires Presente (BAP)}

El programa Buenos Aires Presente se crea en 1999 luego de un fuerte corte de luz de varios días en la zona sur de la ciudad de Buenos Aires que dejó al descubierto que el Gobierno de Buenos Aires no contaba con programas para atender las emergencias de alcance masivo. Esta situación legitimó la creación del BAP que tiene como finalidad

"reforzar los dispositivos de primer nivel de atención social, de la Secretaría de Promoción Social a través de la creación de dos componentes complementarios y asociados a los servicios y programas existentes" (Decreto No 2018, 1999).

Los dos componentes que se mencionan en el decreto citado son el Servicio Social de Atención Telefónica (SAT) ${ }^{5}$ y la Unidad Móvil de Atención Social (UMAS). El SAT implicó la creación de una línea 0800 a través de la cual los vecinos o los propios Sin Techo podían comunicarse gratuitamente con el programa para "denunciar" un caso de emergencia social, propio o ajeno. La línea telefónica funcionaría las $24 \mathrm{hrs}$, los siete días de la semana y la consigna era que toda demanda tenía que tener una respuesta, a partir del trabajo de los diez operadores y los cinco profesionales a cargo del servicio.
La UMAS consistió en la creación de una flota de vehículos para facilitar el traslado de los funcionarios al lugar de donde partió el llamado telefónico y la realización de recorridos permanentes para estar en contacto con los Sin Techo, acercándoles información sobre las prestaciones y sobre sus derechos, contención profesional, y la provisión de elementos de primera necesidad tales como alimentos, vestimenta y abrigo.

Además de incorporar el servicio de vehículos y el centro de llamadas telefónicas, desde el BAP se realizó la primera experiencia piloto de un parador en el barrio de Palermo. Con la creciente creación de dispositivos de este tipo desde el año 2003, en 2006 se crea una instancia de coordinación que será descripta a continuación.

\section{Coordinación de Paradores}

En el año 2006 se crea la Coordinación de Paradores a partir de la firma de un decreto respondiendo a la necesidad de crear una instancia de dirección, control y coordinación de los distintos paradores $y$, de esta forma, darle más impulso a estos dispositivos.

La ciudad fue incrementando la cantidad de paradores estatales, especificándose de acuerdo a las necesidades concretas de la población en situación de calle. En este sentido, en el año 2006 se crea el primer parador para mujeres, con o sin niños, denominado parador Azucena Villaflor, en homenaje a una de las fundadoras de la Asociación de Madres de Plaza de Mayo, sumándose al parador Retiro, al parador Bepo Ghezzi y al Centro de Noche Costanera. Estos últimos tres paradores solo reciben a hombres.

La diferencia entre un parador y un hogar de tránsito es que el primero no reserva vacantes o plazas sino que se cubre por demanda espontánea. Esto implica que las personas deben de llegar en un horario para ubicarse en una fila e ingresar si la capacidad del parador lo permite. En el parador las personas solo pueden pernoctar y no es considerado un espacio para trabajar la reinserción en el mercado de trabajo ni para recomponer

\footnotetext{
Como se verá en el punto 3.3, el centro de llamadas telefónicas luego se transforma en un programa independiente del BAP. El programa Buenos Aires Presente sí conservará la flota de vehículos para asistir a las personas en la misma calle.
} 
los lazos familiares, como sí lo es el hogar. En este sentido, según los relatos de los funcionarios entrevistados, los paradores se constituyeron como la primera instancia de atención de la emergencia, como un primer "colchón" de contención social. Si bien la resocialización es tarea de los hogares de tránsito y es abordada a partir de los talleres de capacitación laboral, en las entrevistas realizadas los funcionarios distinguen otra línea de trabajo para la inserción social en los paradores. En este sentido, las expectativas de logros son de menor escala y la inserción social es interpretada por la posibilidad de tomar una ducha, alimentarse y acceder a un espacio donde se pueda iniciar una relación con un equipo profesional. Teniendo en cuenta la visión de los funcionarios, los paradores también estarían trabajando la inserción social de las personas en situación de calle, pero a partir de una expectativa mucho menor que en los hogares.

\section{Línea de Emergencia Social 108}

En enero del año 2006 se crea por decreto la Línea de Emergencia Social 108 que reemplaza a la línea 0800 antes mencionada, la cual era uno de los dos componentes pilares del BAP. La iniciativa de cambiar el 0800 por el 108, según otra de las funcionarias entrevistadas, respondía, por un lado, a "momentos políticos" y a la mayor facilidad que implica recordar el número, en contraposición a la cantidad de dígitos que tiene una línea 0800 y, por el otro, a restringir el alcance territorial a la ciudad de Buenos Aires. De esta forma, se produce un pasaje de responsabilidades y funciones del programa BAP a la Línea 108.

El objetivo de esta línea, según la funcionaria entrevistada, es dar una respuesta a la demanda social dentro de la ciudad:

"ese era el objetivo: informar, derivar, y contener, ya que tenemos profesionales también atendiendo en la línea... O sea, entra la demanda social, se articula con el BAP que va a la calle con las camionetas. Puede llamar un beneficiario directo o indirecto, como por ejemplo un vecino, y ahí se articula con los diferentes profesionales. Luego, los profesionales del BAP evalúan la situación y ahí se deriva a la persona a diferentes lugares: a paradores o a hogares" (entrevista realizada a la por entonces Coordinadora del programa).

Así es como la Línea 108 trabaja en forma coordinada con el programa BAP. Se reciben demandas telefónicas de vecinos o de Sin Techo que son comunicadas al BAP y este último se acerca con su flota de vehículos al lugar para atender la necesidad planteada.

Hogares y paradores: ¿una nueva forma de abordar el problema habitacional?

Si bien la definición de Sin Techo remite a una relación entre el individuo y lo habitacional, la atención a este grupo desde 1997 estuvo a cargo de los organismos abocados históricamente a la asistencia social. Sin embargo, puede afirmarse que desde estos programas se ofrecen prestaciones que dan cuenta de un nuevo tipo de soluciones habitacionales diferenciadas de lo que se implementó históricamente en Argentina. A lo largo del tiempo, las políticas vinculadas a la vivienda asumieron diferentes modalidades, algunas de ellas vinculadas al acceso a la propiedad mediante subsidios para la autoconstrucción, la entrega de casas terminadas, los loteos económicos y el otorgamiento de créditos de gran alcance en la población. La aparición de los hogares de tránsito y de los paradores representa una nueva forma de concebir y atender la cuestión habitacional.

Ambas instancias de hospedaje, los "hogares" y los "paradores", a pesar de sus diferencias, pueden pensarse como un nuevo tipo de prestaciones tendientes a compensar el problema habitacional y cuentan con características novedosas en relación a las políticas habitacionales tradicionales: son temporarias, ya que tanto los hogares como los paradores son por tiempo limitado (en los paradores la estadía se renueva diariamente; en los hogares, los períodos son de hasta seis meses); son instancias de hospedaje colectivo, es decir que se comparten los espacios con otros beneficiarios; y son ámbitos que cuentan con la presencia permanente de equipos profesionales conformados por psicólogos y trabajadores sociales, quienes deben aprovechar la situación para derivar a las personas a otros organismos públicos que puedan atender los problemas específicos de cada caso. Una de 
las derivaciones más frecuentes es a la oficina de entrega de subsidios habitacionales, en la cual el gobierno de la ciudad otorga partidas de dinero a grupos familiares que prefieren vivir en hoteles. Esta prestación, al igual que los paradores y los hogares, también es por un tiempo determinado, es decir, que no se extiende a largo plazo; la diferencia es que los hoteles sí con espacios de albergue individualizados, esto es, no colectiva, y que los beneficiarios de los subsidios deben sortear entrevistas con los equipos profesionales que gestionan los recursos.

Por lo dicho anteriormente, el surgimiento de los hogares y los paradores pueden ser pensados como una nueva forma de asistir los problemas habitacionales de la población. Por otro lado, parece paradójico que la definición del concepto Sin Techo esté claramente teñida por el aspecto habitacional y que, sin embargo, los organismos públicos abocados a atender a los Sin Techo no sean los que ya cuentan con una trayectoria de trabajo en problemas habitacionales, sino más bien en la asistencia social de los problemas urgentes.

A continuación se dará cuenta de qué formas ciertas categorías creadas para pensar a las personas que viven en las calles entran en crisis a partir de la experiencia acumulada desde 1997 por los funcionarios que trabajan en los distintos programas sociales.

\section{Debates en torno a la atención de las perso- nas Sin Techo}

El trabajo realizado por los funcionarios en forma diaria desde 1997 y la generación de un mayor conocimiento de las características de la población que vive en la calle puso bajo cuestionamiento ciertas categorías como Sin Techo y cronicidad.

El debate existente en los programas apunta a proponer el término "personas en situación de calle" como el más indicado para referirse a las personas que viven en la vía pública. Las primeras preguntas que surgen son: ¿qué diferencias existen entre el concepto "Sin Techo" y "personas en situación de calle"?; ¿cuáles son las razones de este cambio?; ¿qué connotaciones tiene el nuevo término que el concepto Sin Techo no refleja?

Si bien aún no existe un consenso teórico acerca del uso de los conceptos más apropiados dentro de los programas, la funcionaria encargada de la coordinación del Parador Bepo Ghezzi confirmó durante el trabajo de campo realizado en 2008 que Sin Techo es un concepto que está en debate.

"Mirá, no hay una definición acordada acá por el equipo (de trabajo)... Por lo general nuestro término es situación de calle, porque nos parece mucho más amplio por lo que puede englobar. Porque la situación de calle no implica el no Techo, o solo lo habitacional. Digo, los casos que te fui comentando puede tener un techo, hay otras cuestiones que entran en juego para que el tipo esté acá (en el parador). En principio nos parece que situación de calle incluye más la heterogeneidad, la cosa causal de lo que implica estar en calle, que no necesariamente se relaciona con la carencia habitacional"6.

En el testimonio de la funcionaria entrevistada se plantea que la definición de Sin Techo antes mencionada remite solo a una característica de la persona que vive en la calle. Según la entrevistada, reducir el concepto a una carencia habitacional termina dando poca cuenta de la complejidad real de las situaciones que vive la población que está atravesando esta realidad. En este sentido, la fractura de las redes familiares/afectivas, la entrada a las adicciones, el deterioro físico y psíquico y el vínculo con el mundo laboral son otras variables que deberían ser tenidas en cuenta a la hora de pensar la situación de calle. De esta forma, la intención final de la utilización del término "persona en situación de calle" hace referencia a la complejidad del problema por el que atraviesa este grupo y a dimensiones que los programas deben dar cuenta en su trabajo cotidiano con esta población, aún no plasmadas en el desarrollo de conceptos teóricos elaborados por los propios programas del GCBA.

Siguiendo con el relato de la funcionaria, el término Sin Techo intenta homogenei-

Entrevista realizada a la coordinadora del Parador Bepo Ghezzi, en septiembre de 2008. 
zar a partir de la variable habitacional y en el trabajo cotidiano desde los programas se hace presente continuamente la heterogeneidad de la población que vive en la calle:

"Obviamente que al ser una población heterogénea, hay una diferencia de edad, de clase, de causa y consecuencia de por qué está en calle, del tiempo que lleva en calle, de conocimiento del sistema (de redes institucionales) y todo lo que se te puede ocurrir... Pero no pueden reconocer que están en calle como el otro y vienen con esto de que el otro es un delincuente... (Para nosotros) hoy está igual que el otro".

En este fragmento la coordinadora del parador Bepo Ghezzi hace referencia por un lado a la diversidad de historias y trayectorias presentes en las personas en situación de calle, pero también a la imposibilidad de reconocerse entre sí por parte de los propios beneficiarios. En este sentido, continuamente, existe un intento de diferenciación entre las personas en situación de calle y de mostrarse a sí mismos como diferentes al imaginario social que existe en torno a este grupo, el cual los vincula con la mendicidad, la pereza, la suciedad, la delincuencia, entre otros posibles estereotipos. La nueva concepción "persona en situación de calle" pretende dar cuenta de esta diversidad sin perder de vista que existe una situación que unifica a las historias, al menos en el presente, y que debe ser atendida.

Finalmente, la nueva concepción que está siendo debatida va unida a la revisión de ciertas categorías que nacieron con la creación en 1997 del programa Sin Techo. En este sentido, términos como "cronicidad" comienzan a ponerse en tela de juicio.

La categoría cronicidad representó en el año 1997 la división de la población que vive en la calle en dos grandes grupos: los casos irrecuperables y los casos resociabilizables. Un caso es diagnosticado como crónico, es decir como irrecuperable, cuando la persona se encuentra viviendo en la calle hace dos años o más. De esta forma, el tiempo transcurrido en la vía pública fue demarcado como un factor determinante a la hora de pensar el tipo de tratamiento que recibiría el caso. La experiencia de trabajo con esta población después de doce años (1997-2009) arrojó que la complejidad es mayor $y$, de esta forma, también entra en debate qué se entiende por cronicidad más allá de la variable "tiempo", que antes pretendía explicarlo todo.

Los funcionarios consultados durante el trabajo de campo intentaron abrir el debate acerca de los alcances y límites de la categoría cronicidad y enriquecer el concepto para poder abordar de una forma más aproximada a la población beneficiaria. Los funcionarios entrevistados coincidieron en que existía la necesidad de complejizar el análisis de la problemática y que ellos observaban que había otros perfiles dentro de la población de calle. En este sentido, ellos interpretan que sí existe el caso crónico, el cual es imposible de ser reinsertado en la sociedad por el avanzado proceso de deterioro psicofísico y la ruptura de vínculos sociales/laborales. Pero, a su vez, el mismo equipo de profesionales intenta dar cuenta de un nuevo perfil de personas en situación de calle: los crónicos del sistema. Este nuevo concepto estaría dando cuenta de las personas que concurren a un parador y que no son autoválidos, es decir, que han perdido la autonomía para poder construir su propio destino y necesitan la red de recursos institucionales para poder reproducir su orden cotidiano. De esta forma, esta nueva interpretación de la realidad social implica dar cuenta de un nuevo perfil de crónicos, que se encontraría en un punto intermedio entre aquellos casos crónicos que ni siquiera aceptan la asistencia por parte de los programas sociales del GCBA y los resociabilizables que comúnmente se encuentran en los hogares, espacios en los cuales se intenta la reinserción de las personas en el mercado laboral y en las redes familiares (si es que las hay).

Por lo desarrollado anteriormente, tanto la categoría Sin Techo como cronicidad son debatidas fuertemente porque terminan invisibilizando otros aspectos de la problemática. A su vez, la estadística elaborada por el mismo gobierno da cuenta de esas otras variables que no se han tenido en cuenta a la hora de conceptualizar a la población beneficiaria.

Entrevista realizada a la coordinadora del Parador Bepo Ghezzi, en septiembre de 2008. 
Personas que viven en la calle: una problemática más allá de lo habitacional

El Gobierno de la Ciudad de Buenos Aires (GCBA) a partir de 1997 comenzó a elaborar información estadística que da cuenta de distintas dimensiones que permiten reconstruir el perfil de la población en situación de calle.

Como se mencionó anteriormente, la definición de las personas beneficiarias desde los programas del GCBA remite a la relación entre el individuo y la vivienda. Sin embargo, la estadística elaborada por el mismo gobierno refleja otras dimensiones sociodemográficas que describen en una forma más integral la problemática de las personas que viven en la calle. A continuación se mostrarán solo algunas de las dimensiones que visibilizan otras aristas de un mismo problema y que deberían ser tenidas en cuenta desde la gestión pública.

Evolución de la cantidad de personas viviendo en las calles de la ciudad de Buenos Aires

La primera pregunta que surge cuando se intenta describir las características de la población que vive en las calles es cuántas personas se encuentran en esta situación. Desde el año 1997 el GCBA realiza casi en forma anual conteos en toda la ciudad. Si bien estos conteos tienen sus alcances y limitaciones, es importante rescatar que se realizan desde un principio bajo la misma modalidad con lo cual es posible la comparabilidad y reconstruir la evolución de la cantidad de personas viviendo en las calles de la ciudad de Buenos Aires a lo largo del tiempo.
Si bien no se conocen con exactitud las causas del incremento o de la baja de la cantidad de personas en situación de calle de un año a otro, pueden plantearse hipótesis explicativas. En 1997 en la ciudad de Buenos Aires se registraron 1.085 personas (ver Gráfico $\mathrm{N}^{\circ} 1$ ), lo cual en aquel momento resultó una cifra alarmante. En los dos conteos siguientes, 1998 y 2000, la tendencia fue a la baja y podría explicarse a partir del incremento de las prestaciones del Gobierno de la Ciudad de Buenos Aires, es decir, de las camas disponibles en los albergues estatales ${ }^{8}$. En el año 2002 y 2004 las cifras trepan nuevamente y puede adjudicarse a la gran crisis 2001-2002 que vivió la Argentina con incrementos en las cifras de desempleo, subempleo, pobreza e indigencia. Luego, en el 2006 se registra una fuerte baja que puede adjudicarse a la recuperación de la industria de la construcción, del sector servi$\operatorname{cios}^{9}$ y de la economía en general. Finalmente, a partir de 2007 se registra el incremento más vertiginoso de personas en situación de calle y podría adjudicarse a dos razones principales: por un lado, los efectos de la crisis internacional y el nuevo aumento del desempleo ${ }^{10}$; por otro, la implementación desde el Gobierno de la Ciudad de Buenos Aires de una fuerte política de desalojos y de represión en la vía pública mediante la Unidad de Control del Espacio Público (UCEP) tras la perspectiva de la limpieza social y del cuidado del ornamento de la urbe a partir de 2008. Si esto fuera así, el gobierno de la ciudad, presidido por Mauricio Macri, estaría generando más Sin Techo mediante los desalojos, convirtiéndolos en flanco de políticas represivas. Cualquiera sea el caso, lo cierto es que en mayo de 2009 se registró la mayor cantidad de personas viviendo en las calles desde 1997 en la ciudad de Buenos Aires: 1.950 personas.

8 Cabe aclarar que las únicas personas contabilizadas en la noche de los conteos son las personas adultas que se encuentran literalmente en la calle. De esta forma, quienes se encuentran albergados en hogares o en paradores, no son contabilizados. Tampoco los menores de edad que no se encuentren acompañados por adultos.

9 La industria de la construcción y el sector de servicios informales tales como plomería, electricidad, trabajos de albañilería, entre otros, son las dos principales ramas de actividad que proporcionan trabajos a los sectores más pobres, en general en la informalidad, precariedad y con bajos ingresos.

10 Según el Instituto Nacional de Estadística y Censos (INDEC), la tasa de desempleo en la Argentina aumenta con la crisis internacional pero no en forma desmedida. De esta forma, en el momento en que se realizó el último conteo de Sin Techo (abril de 2009), la tasa de desocupación en la Argentina era de $8.4 \%$ y en la ciudad de Buenos Aires de 6.6\%. Para más información, en el tercer trimestre de 2009 se registró un $9.1 \%$ de desocupación en la Argentina, confirmando el aumento sostenido desde el cuarto trimestre de 2008, cuando se había registrado que la tasa de desocupación ascendía al $7.3 \%$. 
Gráfico $N^{\circ} 1$

Cantidad de personas que viven en las calles de la ciudad de Buenos Aires, según año de conteo 1997-2009

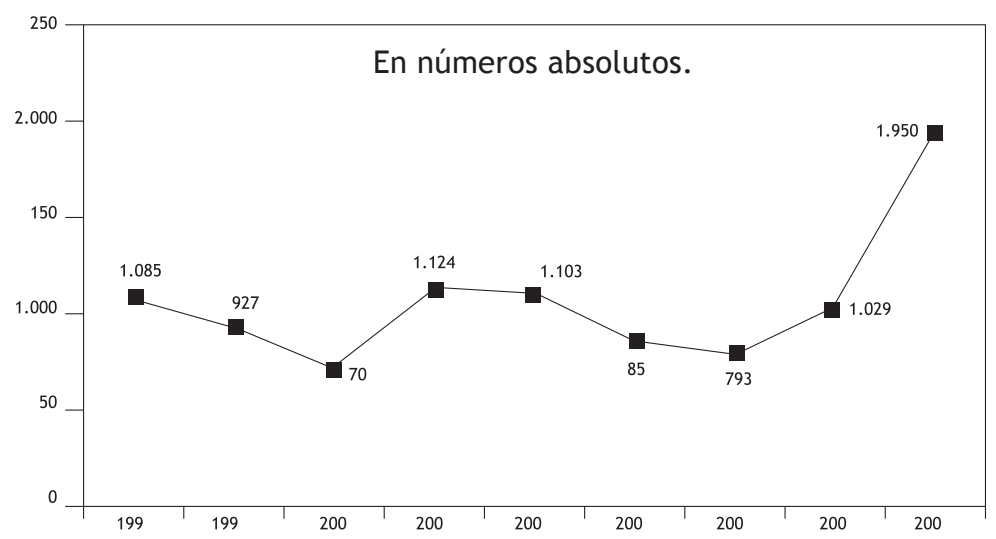

Fuente: Instituto Nacional de Capacitación (INCA). Gobierno de la Ciudad de Buenos Aires.

\section{El género y la edad de las personas que vi- ven en las calles}

Otro de los interrogantes frecuentes es cómo está conformada la población que vive en la calle, teniendo en cuenta la cantidad de hombres y la cantidad de mujeres que componen este grupo. El conteo realizado en 2007 en la ciudad de Buenos Aires arrojó que el $73 \%$ de las personas en situación de calle eran hombres y que el $12 \%$ eran mujeres. Pero como el conteo se realiza a la noche y muchas veces desde los vehículos no puede observarse el género de las personas debido a que están cubiertas por frazadas, en el $15 \%$ de los casos no supo distinguirse si eran hombres o mujeres. Si se calcula nuevamente el porcentaje dejando de lado ese $15 \%$ y tomando como referencia un nuevo $\mathrm{N}$ total, los cálculos indican que el $86 \%$ serían varones y el $14 \%$ serían mujeres. De esta forma, la tasa de masculinidad en la población que vive en las calles es sumamente marcada. Cabe mencionar que situaciones similares han sido registradas en otras ciudades latinoamericanas como Belo Horizonte (Ferreira, Frederico Poley Martins, 2006), Santiago de Chile (Habitando la calle. Catastro Nacional de Personas en Situación de Calle, 2005) y el Distrito Federal en México (Censo de personas en situación de calle de la Ciudad de México "Tú también cuentas", 2008-2009).
La mayoría tan marcada de hombres viviendo en las calles podría explicarse a partir de variables culturales y políticas. En este sentido, diversos estudios con perspectiva de género (Fuller, 2001) ${ }^{11}$ enfatizan en la existencia de mandatos socioculturales a los cuales los varones deben responder y algunos de ellos están vinculados con la gestión de soluciones ante los problemas sin recurrir a la ayuda de otros. En este contexto, la mayor apertura de las mujeres para tejer redes sociales podría explicar esta diferencia tan marcada en todas las ciudades relevadas hasta el momento. Por otro lado, los Estados en general tienden a tener una mayor cobertura de programas sociales de contención para las mujeres, sobre todo si son madres y pobres. En este sentido, la mayor presencia de políticas sociales para ellas y ciertos aspectos culturales podrían explicar por qué la gran mayoría de las personas que viven en la calle son hombres.

En cuanto a la composición etaria en la ciudad de Buenos Aires puede decirse que la mayor cantidad de personas en situación de calle son adultos intermedios, es decir, que no son ni jóvenes ni de la tercera edad. En este sentido, el rango de edad con más casos fue de 31 a 55 años, con el 43\% (ver Gráfico N 2). Estos datos deberían ser tenidos en cuenta a la hora de diseñar e implementar políticas públicas.

FULLER, N. (2001), "Cambios y continuidades en la identidad masculina. Varones de Lima, Cuzco e Iquitos". En La Salud como derecho ciudadano. Memoria del VI Congreso Latinoamericano de Ciencias Sociales y Salud. Perú. 10-13 de junio. 


\section{UNIVERSIDAD CATÓLICA DE TEMUCO}

\section{Gráfico $N^{\circ} 2$}

Población en situación de calle según edad en la ciudad de Buenos Aires, 2007.

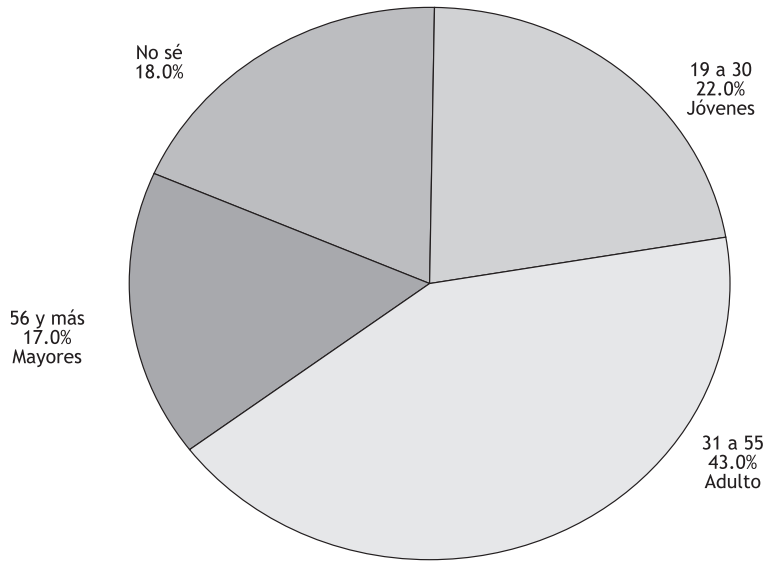

Fuente: Instituto Nacional de Capacitación (INCA). Gobierno de la Ciudad de Buenos Aires.

\section{Personas en situación de calle y migraciones}

Las personas que viven en las calles de la ciudad de Buenos Aires en su mayoría provienen de otras provincias e, incluso, de países limítrofes. Según una encuesta realizada en 2008 (Subsecretaría de Fortalecimiento Familiar y Comunitario, 2008) solo el $31 \%$ de las personas en situación de calle es oriundo de la ciudad de Buenos Aires. La mayoría de los encuestados proviene de provincias del interior del país (34\%), sin contar a la provincia de Buenos Aires. El 22\% proviene de la provincia de Buenos Aires, el $11 \%$ de países limítrofes y el $2 \%$ de países no limítrofes con Argentina (ver Gráfico $\mathrm{N}^{\circ} 3$ ). Por lo tanto, puede afirmarse que los procesos migratorios son una variable muy presente en la población que vive en la calle.

Los procesos migratorios deberían de tenerse en cuenta desde la gestión pública elaborando programas que atiendan a los migrantes y que intenten facilitar la integración de esta población en redes formales y firmes con todos los beneficios que ellas proveen.

\section{Gráfico $N^{\circ} 3$}

Personas en situación de calle según lugar de origen. Ciudad de Buenos Aires, 2008.

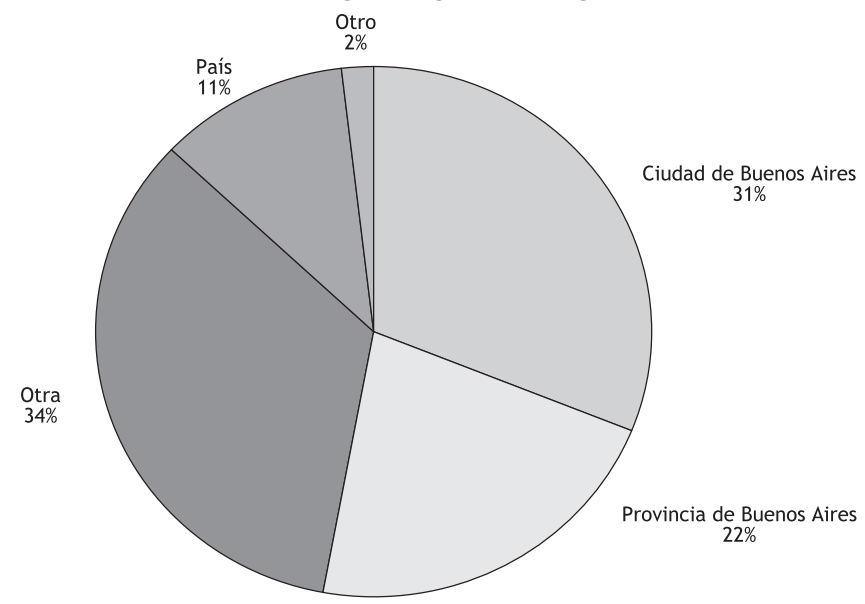

Fuente: Subsecretaría de Fortalecimiento Familiar y Comunitario (2008). 


\section{Personas en situación de calle y barrios de pernocte}

En la ciudad de Buenos Aires, en el año 2007 los resultados del conteo del Gobierno de la Ciudad de Buenos Aires son contundentes: la mayor concentración de personas Sin Techo se registró, según el ex director del Sistema de Atención Inmediata de la Ciudad, en el área central de la ciudad, conocida como el microcentro, y en los barrios de Montserrat, Congreso, Once y San Cristóbal (Página 12, 06/11/2007), todos ellos próximos al centro. Otros barrios que presentan un alto número de personas viviendo en las calles son Palermo y Recoleta, caracterizados por ser habitados por sectores de poder adquisitivo medio-alto y alto, por ser concurridos por turistas y por concentrar grandes zonas comerciales. Distinta es la suerte en los sectores residenciales apartados del centro y de las grandes zonas comerciales como por ejemplo La Paternal y Villa Devoto en donde no se registra presencia de personas viviendo en la vía pública. De esta forma, la distribución geográfica de las personas que viven en la calle parece estar determinada por la abundancia o escasez de recursos de subsistencia, por "los circuitos del rebusque12” (Página 12, 06/11/2007).

\section{Las personas en situación de calle y su rela- ción con el mundo del trabajo}

Puede afirmarse que en la ciudad de Buenos Aires las personas en situación de calle conviven con el desempleo más fuertemente que el resto de la población: el $29.8 \%$ de las personas que viven en la vía pública dijo encontrarse desocupado, mientras que el desempleo en el resto de la población de la ciudad no alcanzó en todo el 2008 el 7\%. De todas formas, si bien el desempleo en la población que vive en la calle es alto, un aspecto que debe resaltarse es que la mayoría de las personas en esta situación se encuentra activa, es decir, desarrolla una actividad que le proporciona dinero. En este sentido, en la ciudad de Buenos Aires el 39.5\% de los encuestados desarrolla actividades que proporcionan ingresos (Ver Gráfico $\mathrm{N}^{\circ}$ 4) estando ocupados a tiempo completo o subocupados ${ }^{13}$. Finalmente, el $30.4 \%$ se encuentra inactivo ${ }^{14}$.

\section{Gráfico $N^{\circ} 4$}

Personas en situación de calle según situación ocupacional. Ciudad de Buenos Aires, 2008.

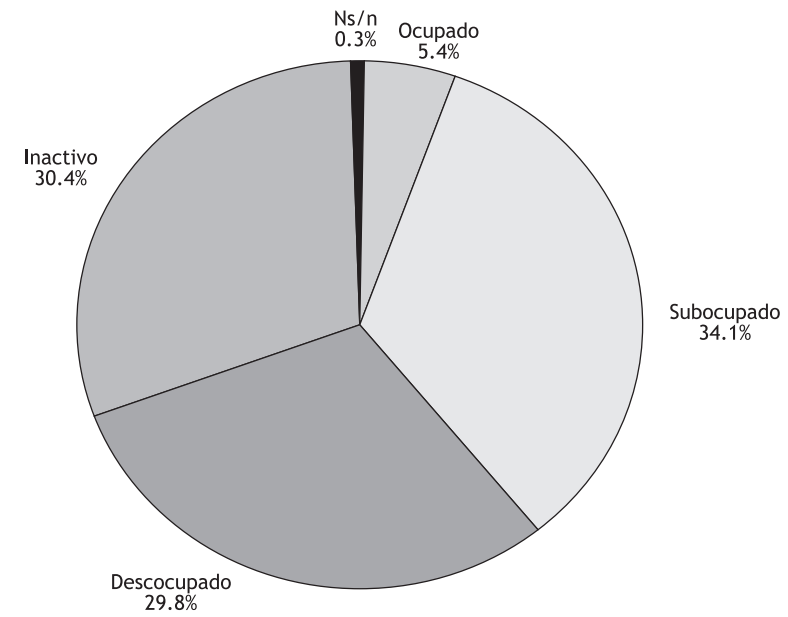

Fuente: Subsecretaría de Fortalecimiento Familiar y Comunitario (2008).

\footnotetext{
12 El término "rebusque" remite a la posibilidad que tienen las personas de encontrar estrategias de supervivencia a partir de los pocos recursos con los que se cuentan.

13 Los subocupados son las personas que trabajan menos de 35 horas semanales y desean trabajar más.

14 La persona inactiva es quien no trabaja, no desea trabajar y no se encuentra buscando un empleo
} 
A modo de conclusión: ¿"Sin Techo" es un concepto apropiado?

Ante la vista de los transeúntes, lo primero que resalta de las personas que viven en la calle es que justamente no poseen una vivienda y esta percepción parece ser la que predominó cuando desde el gobierno se decidió a utilizar el término Sin Techo, el cual tiene como consecuencia la invisibilización de otros aspectos presentes en la población adulta que vive en las calles.

La estadística elaborada por el gobierno porteño da cuenta de otros aspectos centrales de esta población que debería ser tenida en cuenta a la hora de diseñar e implementar políticas sociales. En este sentido, es importante destacar que la gran mayoría de las personas que viven en la calle son migrantes internos, es decir, que provienen de diferentes regiones de Argentina, que se encuentran en una edad en la cual pueden trabajar y que en la mayoría de los casos realizan una actividad que les proporciona ingresos. Estos atributos no aparecen en la conceptualización construida para concebir a esta población ni son suficientemente problematizadas en el momento en que se implementan los programas sociales existentes. Así las cosas, la construcción del concepto Sin Techo determinó que las personas adultas viviendo en la calle estén relacionadas solamente con lo habitacional y las prestaciones que se crearon tendieron a cubrir esta necesidad a partir de los hogares de tránsito y de los paradores. Sin embargo, a pesar de que las cifras dan cuenta de que la mayoría de la población trabaja, ciertos organismos abocados a este tipo de temáticas, como el Ministerio de Trabajo, no se han dado por aludidos ni fueron parte del diseño e implementación de los programas sociales que se crearon. En este sentido, por ejemplo, no se contempló diagramar políticas directas de generación de empleos formales para las personas que viven en la calle con la idea de reinsertar a este grupo en el mercado de trabajo. Por otro lado, tampoco se tuvo en cuenta la posibilidad de implementar políticas de distribución de ingresos para complementar las bajas entradas de dinero que proporcionan las actividades que las personas en situación de calle realizan: venta ambulante, trabajos esporádicos vinculados a la construcción, limpieza de vidrios de coches, entre otros posibles.
Finalmente, puede afirmarse que la relación individuo-vivienda presente en el concepto "Sin Techo" tuvo un peso fuerte a la hora de decidir el tipo de prestaciones que se crearían en el marco del gobierno porteño. En este sentido, los hogares y los paradores surgen como nuevas formas de abordar el problema habitacional, las cuales presentan características particulares: son colectivas, son temporarias y son concebidas como espacios en los que las personas pueden ser reinsertadas socialmente con la ayuda de equipos profesionales.

Cabe preguntarse qué sucederá en caso de que el debate existente al interior de los programas derribe el concepto de "Sin Techo" y de "cronicidad". En caso de que la población beneficiaria sea redefinida como "persona en situación de calle" surgen nuevos interrogantes: ¿podrán incluirse las otras variables visibilizadas por la información estadística? ¿con esta reconceptualización de la población beneficiaria se redefiniría la modalidad de atención a las personas que viven en la calle o se trataría simplemente de una simple corrección política y teórica sin repercusiones reales en la implementación de los programas sociales actuales?

Por los interrogantes planteadas, puede afirmarse que la situación de las personas que viven en la calle ya se encuentra instalada en la agenda de la ciudad, pero que aún es un problema político en construcción.

\section{Bibliografía}

CENSO DE PERSONAS EN SITUACIÓN DE CALLE DE LA CIUDAD DE MÉXICO "TÚ TAMBIÉN CUENTAS", 2008-2009 (2009). Documento elaborado por el Instituto de Asistencia e Integración Social (IASIS); Secretaria de Desarrollo Social y Gobierno del Distrito Federal.

DECRETO No 607/997; Boletín Oficial de la Ciudad de Buenos Aires, N²13; 06/06/1997.

DECRETO N².018; Boletín Oficial de la Ciudad de Buenos Aires; N 803; 22/10/1999.

FERREIRA, F. (2006), “População em situação de rua, vidas privadas em espaços públicos: o caso de Belo Horizonte 1998-2005”. En João Antonio de Paula \& et al. (Comps.), Anais do XII Seminário sobre a Economia Mineira; Minas Gerais. 
HABITANDO LA CALLE. CATASTRO NACIONAL DE PERSONAS EN SITUACIÓN DE CALLE, 2005 (2005); Santiago de Chile, Ministerio de Planificación, Gobierno de Chile. Disponible en: http: //www. fundacionpobreza.cl/Biblioteca/ Archivos/Bajar.asp?Carpeta=POBREZA\&Archiv o=Habitando\%20la\%20calle.pdf

INFORME PRELIMINAR AL DECRETO N 607/997 (1997), titulado "Programa para las personas solas o familias sin techo". Mimeo.

MERKLEN, D. (2000), “La lógica del cazador. Notas sobre sociabilidad y cultura en los asentamientos del GBA hacia fines de los 90". En M. Svampa (Comp.), Desde Abajo. La transformación de las identidades sociales. Editorial Biblos, Buenos Aires.

MORENO, E. (2003), “Desempeño ocupacional: dimensiones en los ciudadanos y ciudadanas habitantes de la calle". En Umbral Científico 002, Bogotá.
PÁGINA 12 (06/11/2007), “En Buenos Aires hay más de mil adultos en situación de calle", Disponible en: http://www.pagina12.com.ar/ diario/ultimas/20-94205-2007-11-06.html

PÉREZ GARCíA, J. (2008), "Derechos de las poblaciones callejeras. Capítulo 31 del diagnóstico de Derechos Humanos del Distrito Federal". Rodríguez G. y Caracol A.C. (Comps.), Diagnóstico de Derechos Humanos del Distrito Federal. Comisión de Derechos Humanos del Distrito Federal México.

SUBSECRETARÍA DE FORTALECIMIENTO FAMILIAR Y COMUNITARIO (2008). "Encuesta a Personas sin hogar alojadas en Hogares de Tránsito y Paradores Nocturnos". Subsecretaría de Fortalecimiento Familiar y Comunitario de la Dirección General de Atención Inmediata del Ministerio de Desarrollo Social del Gobierno de la Ciudad de Buenos Aires. 